\title{
Study of Receptor Status in Carcinoma Breast Patient
}

\author{
Shakera Ahmed ${ }^{1 *}$ \\ Khondker A K Azad' \\ 'Department of Surgery \\ Chittagong Medical College \\ Chittagong, Bangladesh.
}

*Correspondence to:

\section{Dr Shakera Ahmed}

Assistant Professor

Department of Surgery

Chittagong Medical College

Chittagong, Bangladesh.

Mobile : +8801711307876

E-mail: shakeraahmed12@gmail.com

www.banglajol.info/index.php/CMOSHMCJ

\begin{abstract}
Background: Estimation of Estrogen Receptor (ER) Progesterone Receptor (PR) and Human Epidermal Growth Factor Receptor 2 (HER2) is mandatory for choosing treatment options and prognosis of breast cancer patients. This estimation can be done by Immunohistochemistry (IHC) and Fluoroscence In Situ Hybridization (FISH) test. As the tests are expensive many of our patients can't afford to do it. But if we practice doing the test routinely the cost will be reduced and our patients will be benefited. So we underwent a primitive study on receptor status in breast cancer patients. Our goal was to encourage the surgeons to advice receptor status routinely and treat the patients accordingly as patients prognosis will be much more better if they are treated according to their receptor status. Methods: This was a retrospective observational study of 74 breast cancer patients in a private clinic. In this study we have shown their receptor status by IHC and the data were analyzed. Results: Among the 74 patients 32 were ER +ve PR +ve, 11 were ER +ve PR -ve, 02 were ER - ve PR +ve, 29 were ER -ve PR -ve. ER \&/or PR positive patients will be more responsive to hormone therapy. 28 patients were HER $2+v e, 34$ patients were HER2 -ve and 9 were equivocal. HER2 positive patients will be benefited from targeted therapy. Among the patients 11 were triple negative that means they have worst prognosis. Conclusion: As knowing the receptor status in breast cancer help us as a predictive, prognostic and therapeutic indicator, we must advice it routinely and take steps to make it cheaper and easily available.
\end{abstract}

Key words: Receptor; Carcinoma breast; IHC; FISH.

\section{INTRODUCTION}

Incidence of breast cancer is increasing day by day. Breast carcinoma is a heterogeneous disease with a wide spectrum of clinical, pathologic and molecular features ${ }^{1}$. Breast cancer is not only a heterogenous disease but also a hormone dependent disease ${ }^{2}$. The level of ER, PR and HER2 aids in the determination of prognosis and treatment of breast cancer ${ }^{3}$. There are several treatment modalities like surgery, chemotherapy, radiotherapy, hormone therapy etc. The prognosis depends on how accurately we offer treatment to the patient and this depends on doing receptor status or biomarkers in breast cancer patients. Incidence of breast cancer increases with age and elderly patients are more responsive to hormone therapy if they are ER \&/or PR positive. So to know the receptor status is mandatory for treatment of breast cancer. The current standard methodology for measuring ER, PR and HER2 status is Immunohistochemistry (IHC) with additional Fluorescent In Situ Hybridization (FISH) assay to clarify HER2 immunohistochemical status. Although HER2/neu can be detected using many methods, only two are currently approved and recommended for its detection: IHC and $\mathrm{FISH}^{4}$. These tests are very expensive for our patients. Our regular practice of doing the test will reduce the cost. So we underwent a primitive study on receptor status in breast cancer patients. Our aim of the study was to encourage the surgeons to advice biomarkers testing and treat the patients according to the receptor status. 


\section{MATERIALS AND METHODS}

This was a retrospective observational study conducted in Center for Specialised Care \& Research (CSCR) a Private Hospital in Chittagong from March 2008 to Jun 2011. All patients of breast cancer were included who fulfilled the inclusion criteria.

The inclusion criteria was patients with breast cancer, the breast specimen of whom were sent for receptor status by immunohistochemistry.

\section{RESULTS}

Out of 80 patients 74 breast cancer patients could afford immunohistochemistry for knowing their receptor status.

Table 1: Receptor status of the patients.

\begin{tabular}{lcc} 
Groups & Receptor status & No of patients \\
01 & ER+ve,PR+ve & 32 \\
02 & ER+ve, PR-ve & 11 \\
03 & ER-ve, PR+ve & 02 \\
04 & ER-ve, PR-ve & 29 \\
\hline
\end{tabular}

Table 2: HER2 status of the patients.

\begin{tabular}{lcc} 
Groups & HER2 status & No of patients \\
01 & HER2+ve & 28 \\
02 & HER2-ve & 34 \\
03 & Equivocal & 09 \\
\hline
\end{tabular}

HER2 was not done in three patients.

Among the 74 patient 11 were triple negative that is ER, PR and HER2 negative, which means they have the worst prognosis, worst overall and disease-free survival.

\section{DISCUSSIONS}

Biomarkers can be prognostic, predictive or both. Biomarkers testing which should be done are ER, PR, HER2, Ki67 and CK5. Due to unavailability we couldn't do Ki67 and Ck5. ER, $\mathrm{PR}$ is a weak prognostic but strong predictive biomarker. If ER, PR positive (As in $70 \%$ invasive cancer) we can predict that patient will likely be benefited from endocrine therapy like tamoxifen. The overexpression of oncogene HER2 (in 15-20\% invasive cancer) is both a prognostic and predictive biomarker. It is done by immunohistochemistry or FISH method.

Figure 1 : HER2 over expression in breast cancers.

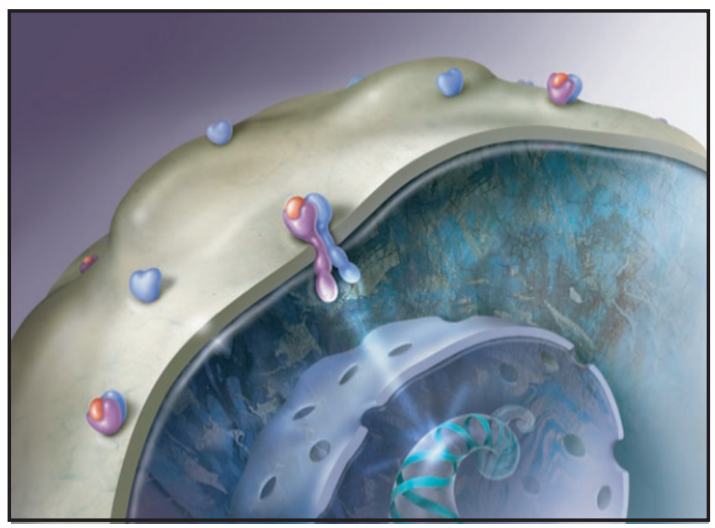

a) Normal HER2 receptors.

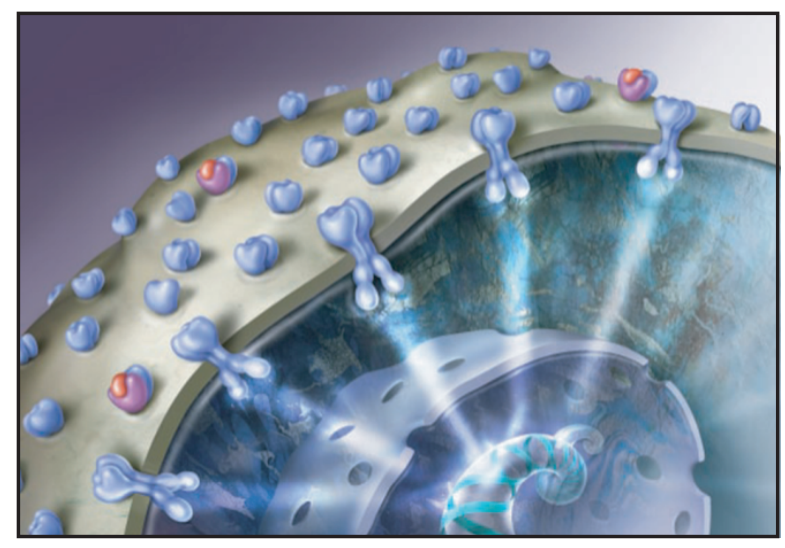

b) Over expressed HER2 receptors.

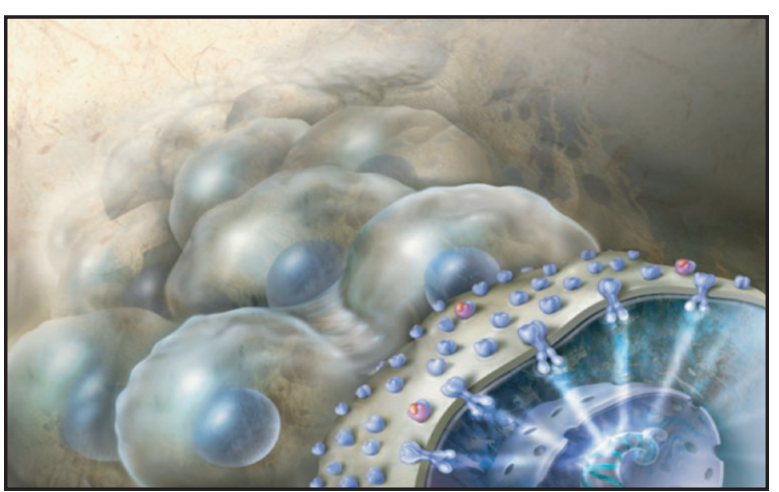

c) Excessive cellular division.

Positive HER2 is associated with bad prognosis ie. higher risk of recurrence and mortality. It also predicts that patient will more likely benefit from targeted therapy.

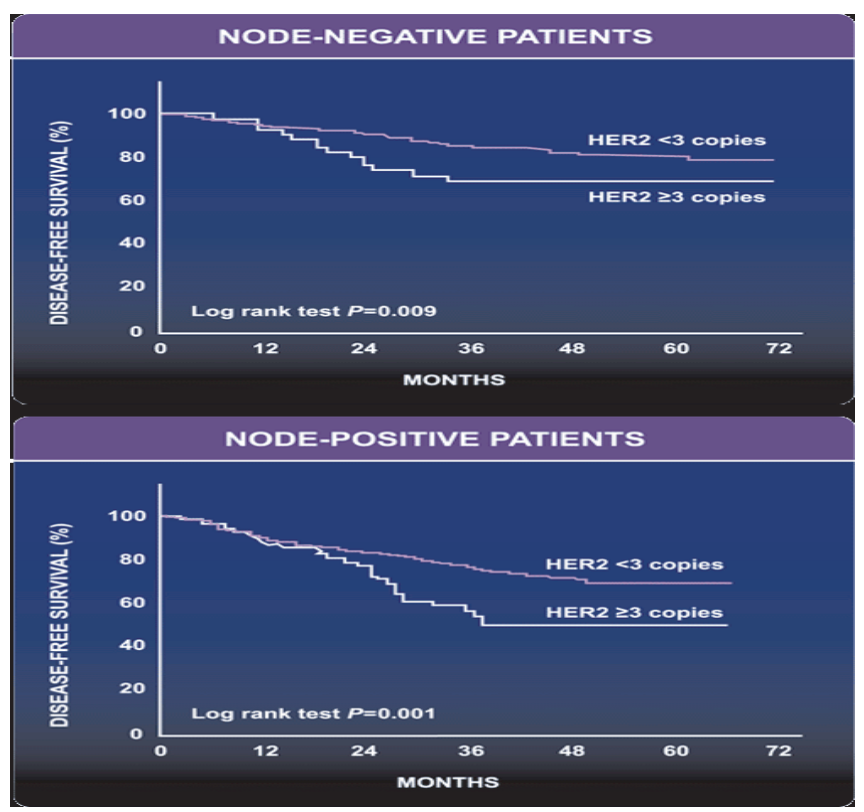

Figure 2 : HER2 as a negative prognostic factor.

HER2 is a negative prognostic factor and is associated with shorter disease free and overall survival. 


\section{ACKNOWLEDGEMENT}

We would like to express our gratitude and thanks to the patients who were convinced to do this expensive test and allowed us to conduct the study.

\section{CONCLUSION}

All women are at risk of breast cancer. We must adopt newer facilities to improve our service. Biomarkers have both prognostic and predictive value and help in choosing treatment options. So it should be practiced routinely. But it is very much expensive in respect to our context so need government and private support.

\section{DISCLOSURE}

Both the authors declared no competing interest.

\section{REFERENCES}

1. Carol A, Parise, Vincent Caggiano. Breast Cancer Survival Defined by the ER/PR/HER2 Subtypes and a Surrogate Classification according to Tumor Grade and Immunohistochemical Biomarkers. Journal of Cancer Epidemiology. 2014. Article ID 469251:11.

2. Ahoua B E, Nguiessan A A, Baumaney S K, et al. Immunohistochemical determination of estrogen receptors in breast cancer: Relationship with clinicopathological factors in 302 patients in Ivory Coast. BMC Cancer. 2017; 17:115.

3. Paul Roepman, Hugo M, Horlings et Al. Microarray-Based Determination of Estrogen Receptor, Progesterone Receptor and HER2 Receptor status in Breast Cancer. Clinical Cancer Research. November 3. 2009.

4. Dana Carmen Zaha. Significance of immunohistochemistry in breast cancer. World Journal of Clinical Oncology. Additional article information. 